\title{
LISTA PRELIMINAR COMENTADA DE LAS “CIGARRITAS” (INSECTA: HEMIPTERA: CICADELLIDAE) DE CHANCHAMAYO Y SATIPO, PERÚ
}

\section{ANNOTATED PRELIMINARY LIST OF THE “LEAFHOPPERS” (INSECTA: HEMIPTERA: CICADELLIDAE) OF CHANCHAMAYO AND SATIPO, PERU}

\author{
Pedro W. Lozada Robles ${ }^{1}$ y Germán A. Arellano Cruz ${ }^{2}$
}

\begin{abstract}
Resumen
Se presenta una lista preliminar comentada de las "cigarritas" de los Fundos Génova, provincia de Chanchamayo y Santa Teresa, provincia de Satipo, Perú. Un total de 46 especies son reportadas, 28 de la subfamilia Cicadellinae, 9 de la subfamilia Deltocephalinae, 4 de la subfamilia Agalliinae, 1 de la subfamilia Gyponinae, 2 de la subfamilia Ledrinae, 1 de la subfamilia Nioniinae, y 1 de la subfamilia Neobalinae. Todas las especies se encuentran depositadas en la colección del Museo de Entomología de la Universidad Nacional Agraria La Molina (UNALM), Lima, Perú. Las siguientes especies se registran por primera vez para el Perú: Haldorus furcatus Caldwell, 1952, Copididonus vittulatus (Berg, 1884), Erythrogonia incerta Medler, 1963, Scaphytopius sulphureus (Osborn, 1923), Agallia atromaculata Oman, 1938, A. lingula Van Duzee, 1907, Agalliana sticticollis (Stål, 1859) y Nionia major Osborn, 1924.

Palabras clave: Lista Comentada, Insecta, Hemiptera, Cicadellidae, “Cigarritas”, Fundo Génova, Fundo Santa Teresa, Chanchamayo, Satipo, Perú
\end{abstract}

\begin{abstract}
A preliminary annotated list of the leafhoppers collected at Fundo Genova, Chanchamayo province and Fundo Santa Teresa, Satipo province, Peru, is presented. A total of 46 species is reported, 28 in the subfamily Cicadellinae, 9 in the subfamily Deltocephalinae, 4 in the subfamily Agalliinae, 1 in the subfamily Gyponinae, 2 in the subfamily Ledrinae, 1 in the subfamily Nioniinae, and 1 in the subfamily Neobalinae. All the species are deposited in the collection of the Museo de Entomologia of the Universidad Nacional Agraria La Molina (UNALM), Lima, Peru. The following species are reported for the first time from Peru: Haldorus furcatus Caldwell, 1952, Copididonus vittulatus (Berg, 1884), Erythrogonia incerta Medler, 1963, Scaphytopius sulphureus (Osborn, 1923), Agallia atromaculata Oman, 1938, A. lingula Van Duzee, 1907, Agalliana sticticollis (Stål, 1859) and Nionia major Osborn, 1924.
\end{abstract}

Key words: Annotated List, Insecta, Hemiptera, Cicadellidae, "Leafhoppers”, Fundo Genova, Fundo Santa Teresa, Chanchamayo, Satipo, Peru

\section{Introducción}

Las “cigarritas" (Hemiptera: Cicadellidae) son insectos fitófagos de importancia como vectores de patógenos en plantas. Como tales, una serie de especies dañan plantas cultivadas, como por ejemplo Dalbulus maidis (Delong \& Wolcott) (Triplehorn \& Nault, 1985), Cicadulina pastusae Ruppel \& DeLong (Ruppel \& DeLong, 1956), o son sospechosas de transmitir enfermedades como en el género Norvellina Ball (Ghauri, 1987). Por ello, el registro de las especies presentes en un lugar o región determinados reviste especial interés para los entomólogos aplicados. En este sentido, se han publicado una serie de trabajos que compendian las especies de “cigarritas” en regiones, países o cultivos determinados (por ejemplo, Remes Lenicov \& Virla, 1993; DeLong \& Severin, 1949; Brown, 1939; Cavichioli \& Zanol, 1991; Linnavuori, 1968; Muruaga de L'Argentier \& Agostini de Manero, 1995; Nielson
\& Currie, 1962; Fattig, 1955; Lozada, 1992b). En este trabajo, reportamos la presencia de 46 especies de “cigarritas" colectadas en el Fundo Génova en la provincia de Chanchamayo y el fundo Santa Teresa en la provincia de Satipo, pertenecientes al Instituto Regional de Desarrollo Selva de la Universidad Nacional Agraria La Molina, ubicados en el departamento de Junín, Perú.

El objetivo de este trabajo es mostrar los resultados de las determinaciones de las "cigarritas" (Hemiptera: Cicadellidae) de las 503 colectas de insectos realizadas entre los años 1984 y 1987, en el marco de un proyecto más complejo en el que se trató de evaluar especies entomológicas dañinas y benéficas en los cultivos de café (Coffea arabiga), papayo (Carica papaya), piña (Ananas comosus), palto (Persea americana), plátano (Musa paradisiaca) y cítricos (Citrus sinensis) en la zona de Chanchamayo y Satipo. Las cigarritas alcanzan mayor importancia 
como potenciales vectores de virus en el cultivo del papayo en la provincia de Chanchamayo, donde la producción bajó de 60,000 TM en 1978 a 6,000 en 1981, debido al ataque del "virus de la mancha de anillo" (Ring Spot Virus) y al mal manejo agronómico. Hasta la fecha se han determinado 46 especies de más de 120 morfoespecies colectadas, cerca de 70 de ellas no pudieron ser determinada por que se encontró sólo un espécimen o la muestra fue muy pequeña en material colectado durante estos años (Arellano, 2001).

\section{Materiales y métodos}

Área de la colecta

El área de influencia del presente estudio, se localiza al oriente de Lima, en la vertiente oriental de los Andes, entre los 10 grados 15' y los 11 grados 30' de latitud sur y desde los 74 grados 30' a los 75 grados 30' al este de Greenwich, en las provincias de Chanchamayo y Satipo, Departamento de Junín.

La colecta se realizó en dos fundos del Instituto Regional de Desarrollo Selva de la Universidad Nacional Agraria La Molina, El fundo Génova con 577 ha, en el valle de Chanchamayo en la margen izquierda del río, cerca a San Ramón entre los 940 msnm y 1200 msnm. El fundo Santa Teresa con 300 ha, situado en el valle de Río Negro a $9.5 \mathrm{~km}$ de la ciudad de Satipo sobre la Carretera Marginal a 850 msnm desde Octubre de 1984 hasta Agosto de 1987

\section{El método básico de colección}

La evaluación se basó en colecciones periódicas de insectos en las plantaciones de café, papaya, piña, palto, cítricos, plátano, y malezas. Los especimenes del total de la colecta fueron remitidos al Museo de Entomología de la Universidad Nacional Agraria La Molina para su montaje y determinación.

Las colecciones de campo se efectuaron cada dos semanas en los cultivos de café, papaya, palto, cítricos y plátano con las malezas asociadas en el fundo Génova y una semana al mes en el fundo Santa Teresa para la piña, papaya y cítricos. Se completaron 503 colecciones de insectos en total donde se incluyen las cigarritas.

La colecta consistió en la captura adultos, mediante aspiradores y redes entomológicas, en los brotes, hojas, flores, frutos y tallos de las plantas cultivadas. En la hierba, la colección se hizo básicamente con red, realizando batidas de ida y vuelta sobre la maleza

Las plantas de los cultivos a evaluarse fueron escogidas al azar a lo largo de una línea en diagonal que cruzaba el campo en todos los cultivos. Las colectas fueron de una duración de 4 a 6 horas, durante el día en horas de luz y de mayor temperatura, entre las 9 y 15 horas, interdiarias, alternando los cultivos, evaluando a todos en dos semanas. En la maleza la colecta se realizó durante una hora siguiendo la misma ruta una vez concluidas las

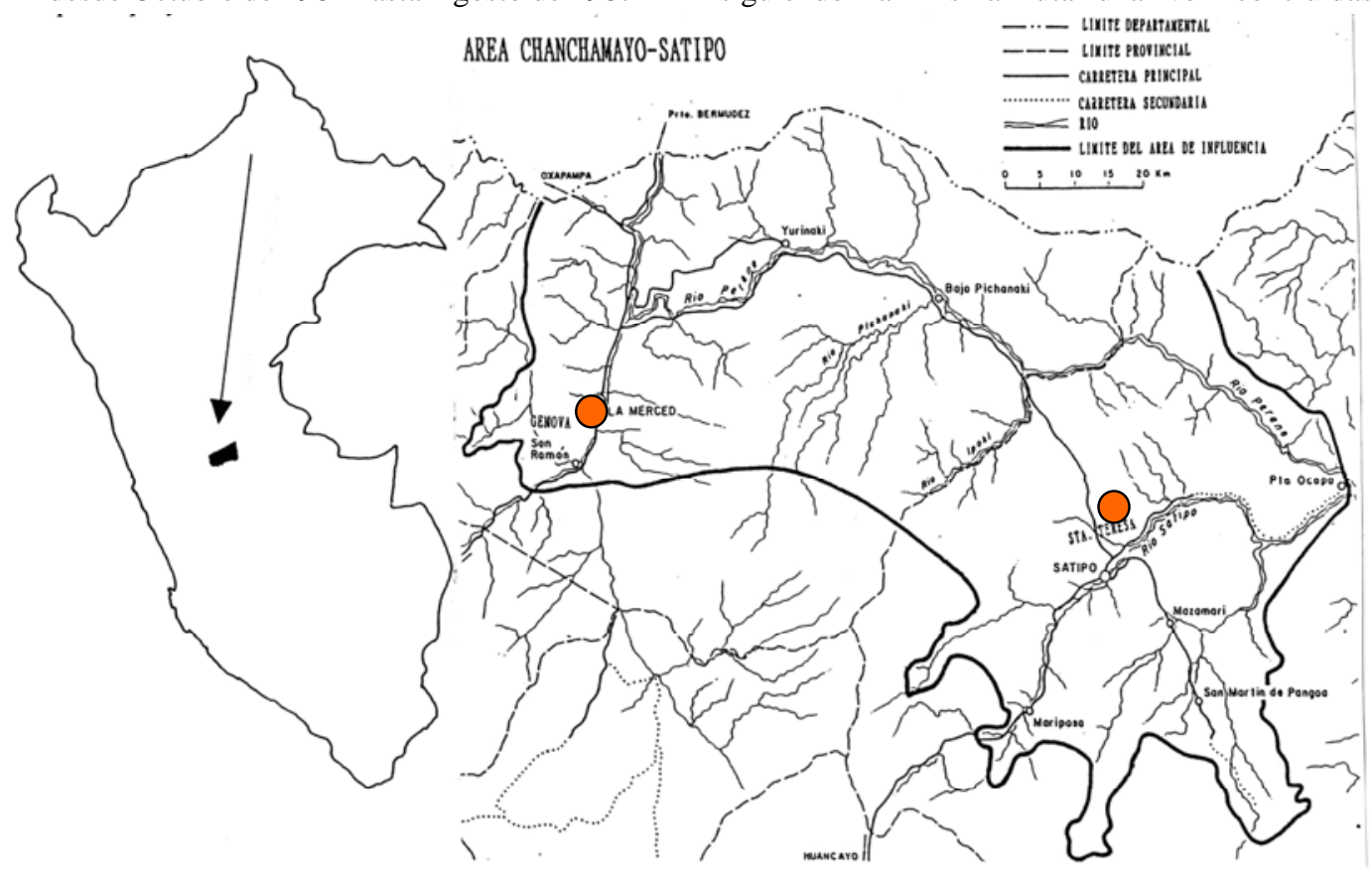

Figura 1. Localización del área de estudio. Zona de influencia de la Carretera Marginal de la Selva entre las ciudades de San Ramón y Satipo en el Departamento de Junín (Arellano, 2001) 
observaciones de las plantas cultivadas.

Durante un año desde Junio de 1986 hasta Junio de 1987, se llevó un registro numérico de los individuos colectados, por morfoespecies y por fecha de las más abundantes y comunes a los cultivos y las malezas. Toda la colecta que llegaba del campo era seleccionada y comparada con una colección de referencia en cajas "Schmidtt" que se conservó en el laboratorio. En ella se mantenían muestras de las morfoespecies identificadas con un código. Los insectos de la Familia Cicadellidae que se presentan en este trabajo se encontraban entre ellos y las observaciones permitieron determinar las fechas en que aparecen con mayor abundancia. Posteriormente el material entomológico de la familia Cicadellidae fue determinado en el Museo de Entomología de la Universidad Nacional Agraria La Molina por el autor principal.

La determinación de las especies

Para la identificación el abdomen de los machos de las "cigarritas" fue disectado y expuesto a una solución al $10 \%$ de hidróxido de potasio en caliente para disolver el contenido abdominal y transparentar las estructuras genitales. Posteriormente, el abdomen fue lavado en agua destilada y guardado en glicerina dentro de un microvial para genitalia. La identificación hasta especie se realizó en base a los caracteres de la genitalia masculina.

\section{Resultados}

\section{Subfamilia CICADELLINAE \\ Tribu PROCONIINI}

Acrogonia terminalis Young, 1968: Se presentó de Febrero a Agosto en la hierba del papayal en el Fundo Génova. Representa el primer registro de la especie con localidad conocida en el Perú. Se distribuye también en Bolivia.

A. virescens Metcalf, 1949: Se presentó también en la hierba del papayal en el fundo Génova. Al igual que la especie anterior, es el primer registro de la especie con localidad conocida en el Perú. Se distribuye también en Guyana, Brasil y Paraguay.

Diestostemma stesilea Distant, 1908: Se le encontró de Abril a Mayo alimentándose de los tallos tiernos de los brotes de los cafetos en el fundo Génova. No ha sido encontrada interiormente con localidad conocida en el Perú. Se distribuye también en Venezuela, Colombia y Bolivia.

Homoscarta irregularis (Signoret, 1855): Se presentó en la hierba del cafetal en el fundo Génova. No ha sido registrada previamente con localidad conocida en el Perú. Se distribuye también en Bolivia y Brasil.

Pseudometopia amblardii (Signoret, 1855): Se presentó de Abril a Junio en los brotes de los cítricos, cafetos y cogollo o meristema terminal del plátano y en la hierba de estos cultivos en el fundo Génova. Es una de las especies más comunes del género en el Perú. Se distribuye también en Colombia, Ecuador, Bolivia, Brasil, Paraguay y Argentina.

Pseudometopia phalaesia (Distant, 1908): Se presentó en los brotes de los cítricos donde se alimentan de los tallos tiernos, al igual que en los paltos y los cafetos, en las hojas de los papayos y en el cogollo y hojas tiernas del plátano en el fundo Génova. También en las malezas de estos cultivos. Junto con la anterior, es una especie común en el Perú. Se distribuye también en Colombia y Bolivia.

Tretogonia cribrata Melichar, 1926: Se presentó de Junio a Setiembre en la hierba de los cítricos y en plantaciones de papaya en el fundo Génova. Es la especie más común del género en el Perú. Se distribuye también en Brasil, Guyana Francesa y Bolivia.

\section{Tribu CICADELLINI}

Catagonalia lunata (Signoret, 1854): Se presentó en los cafetos en el fundo Génova. Es una especie muy común en la Selva peruana. Está limitada a hábitats abiertos con vegetación secundaria. Se distribuye también en Ecuador, Bolivia y Brasil.

Diedrocephala bimaculata (Gmelin, 1789): Conocida anteriormente como $D$. variegata (McKamey, 2007). Se presentó de Mayo a Diciembre en las hojas de los cítricos, cafetos y paltos en el fundo Génova, pero con mayor abundancia en la maleza de estos cultivos. Igualmente, es la especie más común del género en la Selva peruana. De amplia distribución en Centro y Sudamérica.

Erythrogonia aurivagula (Jacobi, 1905): Se presentó en la hierba del paltal en el fundo Génova. Medler (1963) la citó de Perú (Marcapata y Tingo María) y de Bolivia.

E. incerta Medler, 1963: Se presentó en el palto en el fundo Génova. Es primer registro de la especie para el Perú. Medler (1963) la citó de Colombia.

E. sexguttata (Fabricius, 1803): Se presentó abundantemente en la hierba durante todo el año en el fundo Génova. También se registró sobre las hojas de paltos, cítricos, cafetos y en los pecíolos de los papayos. Medler (1963) la citó de Argentina, Brasil, Guyana Francesa, Guyana, Venezuela, Perú y Bolivia.

E. socialis Melichar, 1926: Se presentó de Agosto a Setiembre en la hierba de los cafetos. Medler (1963) la citó de Perú (Marcapata) y Bolivia.

Ferrariana trivittata (Signoret, 1854): Es una especie probablemente restringida a Selva Central. Se distribuye también en Costa Rica, Panamá, Colombia, Bolivia, Brasil, Paraguay y Argentina.

Fusigonalia optata Young, 1977: Se presentó con mayor incidencia en Mayo en la hierba del cafetal en el fundo Génova. Ha sido reportada por Young (1977) de Chanchamayo y Tingo María. Se distribuye también en Bolivia.

Hortensia similis (Walker, 1851): Se presentó de Setiembre a Mayo en la hierba de las plantaciones de 
palto, cafeto y cítricos en el fundo Génova. También en las hojas de cafetos y paltos. Es una especie muy común en toda la América Tropical. En el Perú se distribuye en Costa, Sierra y Selva, asociada al menos a gramíneas. Lozada (1992b) la reportó de “maicillo" en la zona de Jenaro Herrera, departamento de Loreto, Perú.

Juliaca huasima Young, 1977: Se presentó durante todo el año en los cafetos y la maleza de este cultivo en el fundo Génova. Ha sido citada en Chanchamayo y Tingo María por Young (1977). No ha sido informada su presencia en ningún otro país.

Macugonalia chanchama Young, 1977: Se presentó durante todo el año en los cítricos, paltos, papayos macadamia, cafetos y en la hierba de las plantaciones de papayo, cafeto y palto en el fundo Génova. Ha sido reportada de Chanchamayo (Young, 1977), donde es una especie muy común en zonas alteradas. informada su presencia en ningún otro país.

M. moesta (Fabricius, 1803): Se presentó de Julio a Setiembre en la hierba de las plantaciones de papaya y palto en el fundo Génova. Es la especie más común del género en la Selva peruana, donde puede ser encontrada en zonas alteradas. Se distribuye también en Guyana, Guyana Francesa, Brasil, Ecuador y Bolivia.

Microgoniella pudica (Fabricius, 1803): Se presentó durante todo el año en las hojas de los cafetos, alimentándose de los brotes en el fundo Génova; en menor frecuencia en plátano y cítricos. También se presentó en la hierba de estas plantaciones. Es la especie más común del género en la Selva peruana. El autor principal (Lozada, 1992a) describió Microgoniella minka de la selva del departamento de Madre de Dios, Perú. Se distribuye también en Colombia, Bolivia y Brasil.

Oragua partitula (Jacobi, 1905): Se presentó en la hierba del cafetal en el fundo Génova. Parece ser una especie muy común en la Selva peruana. Se distribuye también en Bolivia.

Plesiommata mollicella (Fowler, 1900): Se presentó en la hierba del papayo en el fundo Génova. Es una especie muy común en zonas alteradas en la Amazonía peruana. Lozada (1992b) la reportó de "maicillo" en la zona de Jenaro Herrera, departamento de Loreto, Perú. Se distribuye en Centro y Sudamérica.

Ramosulus corrugipennis (Osborn, 1926): Se presentó durante todo el año en las hojas tiernas y en los brotes de los cafetos en el fundo Génova. En forma ocasional en el plátano. Es una especie común en zonas alteradas de Selva Central. Se distribuye también en Brasil, Ecuador y Bolivia.

Scopogonalia subolivacea (Stål, 1862): Se presentó de Mayo a Diciembre en la hierba de plantaciones de palto en el fundo Génova. Es una especie común en zonas alteradas en la Selva Central.
Se distribuye también en Bolivia, Brasil, Paraguay y Argentina.

Sibovia huasima Young, 1977: Se presentó de Junio a Setiembre en los cafetos y la maleza de este cultivo en el fundo Génova. Young (1977) la reportó de Chanchamayo y Tingo María. Está restringida al Perú.

S. taeniatifrons (Schmidt, 1928): Se presentó de Mayo a Agosto en paltos y la hierba de plantaciones de cafeto y papayo en el fundo Génova. Young (1977) reportó que colectó esta especie en Chanchamayo en compañía del Ing. Juan Salazar. Se distribuye también en Colombia y Ecuador.

Tylozygus geometricus (Signoret, 1854): Se presentó de Marzo a Agosto en la hierba del papayo, con mayor abundancia en Mayo en el fundo Génova. Es una especie común en Selva Central. Se distribuye desde el sur de Estados Unidos de Norteamérica hasta el sur de Brasil.

\section{Subfamilia DELTOCEPHALINAE \\ Tribu DELTOCEPHALINI}

Haldorus furcatus Caldwell, 1952: Se presentó en la hierba del cafeto en el fundo Génova.Primer registro de la especie para el Perú. Está presente en Puerto Rico (Linnavuori, 1959).

Planicephalus flavicosta (Stål, 1860): Se presentó de Marzo a Agosto en los cafetos, cítricos, papayos y en la hierba de estos cultivos en el fundo Génova. Es una especie ampliamente distribuida en Centro y Sudamérica. Lozada (1992b) la reportó como Planicephalus sp. en "maicillo" en Jenaro Herrera, departamento de Loreto, Perú.

Stirellus bicolor (Van Duzee, 1892): Se presentó durante todo el año, con la mayor abundancia en Mayo, en la hierba de las plantaciones de papayo, palto, cítricos y cafeto en el fundo Génova. Es una especie común en zonas alteradas de la Selva Central peruana. Se distribuye además en Norte y Centroamérica, y en Sudamérica en Colombia, Surinam, Brasil y Bolivia.

S. picinus cuneatus Uhler, 1895: Se presentó en la hierba del papayal en el fundo Santa Teresa, Satipo. Lozada (1992b) la reportó de “maicillo” en la zona de Jenaro Herrera, departamento de Loreto, Perú. Se distribuye también en el Caribe, Surinam y Paraguay.

\section{Tribu EUSCELINI}

Copididonus vittulatus (Berg, 1884): Se presentó en el cafeto en el fundo Génova. Primer registro para el Perú. Se distribuye también en Surinam, Brasil y Argentina. Lozada (1992b) reportó C. hyalinipennis (Stål) de "maicillo" en Jenaro Herrera, departamento de Loreto, Perú.

Exitianus obscurinervis (Stål, 1859): Se presentó durante todo el año, con mayor incidencia de Febrero a Julio, en la hierba del papayo en el fundo Génova. El género está representado en Costa, Sierra y Selva. La especie ha sido erróneamente citada de Callao, Perú 
(Linnavuori, 1959), registro que en realidad corresponde a una especie no determinada del género (Lozada, dato no publicado). Se distribuye también en Brasil, Paraguay, Uruguay y Argentina.

Menosoma elegans (Osborn, 1923): Se presentó en la hierba de los cítricos en el fundo Santa Teresa, Satipo. La especie más común del género en la Selva peruana. Lozada (1992b) la reportó de la zona de Jenaro Herrera, departamento de Loreto, Perú. Se distribuye también en Brasil, Bolivia, Paraguay y Argentina.

\section{Tribu SCAPHYTOPIINI}

Scaphytopius (Convelinus) nr. neloricatus (Caldwell, 1923): Se presentó de Noviembre a Mayo en la hierba de los cítricos en el fundo Génova. La especie se distribuye en Puerto Rico (Linnavuori, 1959).

S. (Cloanthanus) sulphureus (Osborn, 1923): Se presentó de Mayo a Agosto en las hojas de cítricos y palto en el fundo Génova. Primer registro de la especie para el Perú. Se distribuye también en Bolivia (Linnavuori, 1959).

\section{Subfamilia AGALLIINAE}

Agallia atromaculata Oman, 1938: Se presentó de Mayo a Agosto , con mayor abundancia en Agosto, en el papayo, cafeto y en la hierba de estos cultivos en el fundo Génova. Primer registro de la especie para el Perú. Metcalf (1966) la citó de Bolivia.

A. depleta Oman, 1933: Se presentó de Mayo a Junio en el fundo Santa Teresa, Satipo. Lozada (1992b) la colectó de “maicillo” y “kudzú” en Jenaro Herrera, departamento de Loreto, Perú. Metcalf (1966) la citó de Guyana, Brasil, Argentina y Venezuela.

A. lingula Van Duzee 1907: Se presentó en la hierba del papayo en el fundo Génova. Primer registro de la especie para el Perú. Metcalf (1966) la citó de México, EEUU y Centroamérica.

Agalliana sticticollis (Stål, 1859): Colectada en una oportunidad en los cafetos en el mes de Mayo. Oman (1934) la citó de Brasil y Argentina. Primera vez que se registra para el Perú, aunque es una especie relativamente común en la Selva peruana.

\section{Subfamilia GYPONINAE}

Gypona glauca (Fabricius, 1803): Se presentó en los meristemos terminales del plátano en el fundo Génova. Una especie común en material de Gyponinae de la Selva peruana. DeLong \& Freytag (1962) la citaron de Brasil.

\section{Subfamilia NIONIINAE}

Nionia major Osborn, 1924: Fue colectada en Chanchamayo en Noviembre de 1971. Primer registro del género y la especie para el Perú. Citada anteriormente sólo de Bolivia (Linnavuori, 1956).
Proranus ghilianii Spinola, 1850: Se presentó de Diciembre a Marzo en papayo en el fundo Génova. Kramer (1966) la citó de Brasil, Bolivia, Venezuela, Colombia y Perú, y Freytag \& Sharkey (2002) la citaron de Colombia.

Xerophloea viridis (Fabricius, 1794): Se presentó de Setiembre a Enero en la hierba del papayo en el fundo Génova. Oman (1936) la citó de Argentina, Chile, Perú, Venezuela y Brasil.

\section{Subfamilia NEOBALINAE}

Calliscarta invita (Nast, 1952): Se presentó en cafeto en el fundo Génova. Freytag (1988) la citó de Perú y Ecuador.

\section{Discusión y conclusiones}

Es la segunda vez que se reportan las “cigarritas” de una región o zona determinadas para el Perú. La primera (Lozada, 1992b) estudió las especies de “cigarritas” presentes en "maicillo” y "kudzú", dos plantas forrajeras, en la zona de Jenaro Herrera en el departamento de Loreto. Una parte de las especies se anotan como nuevos registros para nuestro país, como son: Haldorus furcatus, Copididonus vittulatus, Erythrogonia incerta, Scaphytopius sulphureus, Agallia atromaculata, A. lingula, Agalliana sticticollis y Nionia major. Todas estas especies están presentes en zonas alteradas, donde el bosque primario ha sido talado para utilizar el área como zona cultivada, lo que no significa que determinadas especies estén distribuidas a la vez en estos dos tipos de ambientes. Hortensia similis y el género Exitianus están distribuidos en las tres regiones peruanas: Costa, Sierra y Selva. Todas las especies son mantenidas en la colección del Museo de Entomología de la Universidad Nacional Agraria La Molina (UNALM), Lima, Perú. Es muy necesario mantener especimenes "voucher" de los estudios ecológicos que se realicen (Huber, 1998), especialmente en la Amazonía, donde poco se conoce de su entomofauna, sobre todo la de importancia económica, donde hay que destacar los trabajos realizados en el departamento de Loreto (por ejemplo, Delgado \& Couturier, 2004).

\section{Agradecimientos}

Nuestro más sincero agradecimiento a la Blga. Mg. Sc. Clorinda Vergara por permitir al autor principal la observación y estudio del material de "cigarritas" colectado por el segundo autor en los fundos Génova, provincia de Chanchamayo, y Santa Teresa, provincia de Satipo, bajo su custodia en el Museo de Entomología de la Universidad Nacional Agraria La Molina, Lima - Perú.

\section{Subfamilia LEDRINAE}




\section{Literatura citada}

Arellano G.A. 2001. Evaluación de plagas en Café, Papayo, Piña, Palto, Plátano y Cítricos en Chanchamayo y Satipo. Tesis para optar el grado de Magister Scientiae, especialidad Entomología. Escuela de Post-Grado, Universidad Nacional Agraria La Molina, Lima Perú.

Brown H.E. 1939. An annotated list of the species of Jassinae known to occur in Indiana (Homoptera, Cicadellidae). The American Midland Naturalist. 21(3): 663-673.

Cavichioli R.R. \& Zanol K.M.R. 1991. Espécies de Cicadellinae, Deltocephalinae (Homoptera, Cicadellidae) da Ilha de Maracá, Roraima. Acta Amazonica. 21: 55-61.

Delgado C. \& Couturier. G. 2004. Manejo de Insectos Plagas en la Amazonía: Su Aplicación en Camu Camu. Lima, Instituto de Investigaciones de la Amazonía Peruana (IIAP), Institut de Recherche pour le Dévelopment (IRD), Tarea Asociación Gráfica Educativa.

Delong D.M. \& Freytag P.H. 1962. Studies of the Gyponini (Homoptera: Cicadellidae): The Gypona glauca (Fabricius) complex. Bulletin of the Brooklyn Entomological Society. 57(4): 109-131.

Delong D.M. \& Severin H.H.P. 1949. Characters, distribution, and food plants of leafhopper vectors of virus causing Pierce's disease of grapevines. Hilgardia. 19(6): 171-186.

Fattig P.W. 1955. The Cicadellidae or leafhoppers of Georgia. Emory University Museum Bulletin. 11: 1-68.

Freytag P.H. 1988. Revision of the genus Calliscarta (Homoptera: Cicadellidae: Neobalinae). En: Research in the Auchenorrhyncha, Homoptera: A Tribute to Paul W. Oman. Great Basin Naturalist Memoirs. 12: 67-81.

\& Sharkey M.J. 2002. A preliminary list of the leafhoppers (Homoptera: Cicadellidae) of Colombia. Biota Colombiana. 3(2): 235-283.

Ghauri M.S.K. 1987. Two new species of Norvellina Ball from Ecuador, suspected as vectors of Mycoplasma-like organisms (MLO) (Homoptera, Cicadelloidea, Cicadellidae). Reichenbachia 24(25): 167-170.

Huber J.T. 1998. The importance of voucher specimens, with practical guidelines for preserving specimens of the major invertebrate phyla for identification. Journal of Natural History. 32: 367-385.

Kramer J.P. 1966. A revision of the New World leafhoppers of the subfamily Ledrinae (Homoptera: Cicadellidae). Transactions of the American Entomological Society. 92: 469-502.

Linnavuori R. 1956. Neotropical Homoptera of the Hungarian National Museum and some other European museums. Annales Entomologici Fennici. 22(1): 5-35.

1959. Revision of the Neotropical Deltocephalinae and some related subfamilies. Annales Zoologici Societatis Zoologicæ Botanicæ Fennicæ "Vanamo". 20(1): 1-370.
1968. Contribución al conocimiento de la fauna colombiana de cicadélidos. Agricultura Tropical 24(3): 147-156.

Lozada P.W. 1992a. Una nueva especie de Microgoniella Melichar de Perú (Homoptera: Cicadellidae). Bulletin de la Société Entomologique de France. 97(2): 167-169.

1992b. Notas sobre Cicadellidae (Homoptera) en plantas forrajeras de Loreto, Perú. Revista peruana de Entomología. 35: 24-26.

McKamey, S.H. 2007. Taxonomic Catalogue of the Leafhoppers (Membracoidea). Part 1. Cicadellinae. Memoirs of the American Entomological Institute. 78: 1-394.

Medler J.T. 1963. A review of the genus Erythrogonia Melichar (Homoptera: Cicadellidae). Miscellaneous Publications of the Entomological Society of America. 4(1): 3-30.

Metcalf Z.P. 1966. General Catalogue of the Homoptera. Fascicle VI, Cicadelloidea. Part 14: Agalliidae. Washington, D.C., United States Department of Agriculture, Agricultural Research Service.

Muruaga De L'Argentier S. \& Agostini De Manero E. 1995. Contribución al estudio de las especies de Cicadellidae (Homoptera) presentes en cultivo de poroto en Jujuy (Argentina). Revista de la Sociedad Entomológica Argentina. 54(1-4): 77-82.

Nielson M.W. \& Currie W.E. 1962. Leafhoppers attacking alfalfa in the Salt River valley of Arizona. Journal of Economic Entomology. 55(5): 803-804.

Oman P.W. 1934. South American leafhoppers of the genus Agalliana. Revista de Entomología. 4(3): 333-340. 1936. South American leafhoppers of the genus Xerophloea Germar (Hom.). Revista de Entomología. 6(3-4): 394-401.

Remes Lenicov A.M.M. \& Virla E.G. 1993. Homópteros auquenorrincos asociados al cultivo de trigo en la República Argentina. I. Análisis preliminar de la importancia relativa de las especies. Studies on Neotropical Fauna and Environment. 28: 211-222.

Ruppel R.F. \& Delong D.M. 1956. A new species of Cicadulina (Homoptera: Cicadellidae) from Colombia. Bulletin of the Brooklyn Entomological Society. 51(3): 82-84.

Triplehorn, B.W. \& L.R. Nault. 1985. Phylogenetic classification of the genus Dalbulus (Homoptera: Cicadellidae), and notes on the phylogeny of the Macrostelini. Annals of the Entomological Society of America. 78: 291-315.

Young D.A. 1968. Taxonomic study of the Cicadellinae. Part I. Proconiini. Bulletin of the United States Natural History Museum 261: 1-287. 1977. Taxonomic study of the Cicadellinae. Part II. New World Cicadellini and the genus Cicadella. North Carolina Agricultural Experiment Station Technical Bulletin. 239: 1-1135..

\footnotetext{
${ }^{1}$ Departamento de Entomología, Museo de Historia Natural, Universidad Nacional Mayor de San Marcos (UNMSM), Av. Arenales 1256, Apartado 14-0434, Lima 14, Perú. Correo electrónico: plozada21@gmail.com, plozada@daad-alumni.de

${ }^{2}$ Laboratorio de Ecología de Insectos, Departamento de Biología, Universidad Nacional Agraria La Molina. Av. La Molina S/N, Lima 12 PERÚ. Correo electrónico: acg@lamolina.edu.pe
} 